\title{
A fixed point approach to the solution of singular fractional differential equations with integral boundary conditions
}

\author{
Kalaivani Chandran ${ }^{1}$, Kalpana Gopalann ${ }^{1 *}$, Sumaiya Tasneem Zubair ${ }^{1}$ and Thabet Abdeljawad ${ }^{2,3,4^{*}}$ (D)
}

\author{
"Correspondence: \\ kalpanag@ssn.edu.in; \\ tabdeljawad@psu.edu.sa \\ 'Department of Mathematics, Sri \\ Sivasubramaniya Nadar College of \\ Engineering, Kalavakkam, Chennai, \\ 603 110, India \\ ${ }^{2}$ Department of Mathematics and \\ General Sciences, Prince Sultan \\ University, P.O. Box 66833, Riyadh \\ 11586, Saudi Arabia \\ Full list of author information is \\ available at the end of the article
}

$$
\begin{aligned}
& \text { Abstract } \\
& \text { In this article, we first demonstrate a fixed po } \\
& \text { the setting of controlled } b \text {-Branciari metric } \\
& \text { - Thereafter, we specifically consider a fol } \\
& \text { a singular fractional differential equation } \\
& \qquad{ }^{c} D^{\alpha} v(t)+h(t, v(t))=0, \quad 0<t<1, \\
& v^{\prime \prime}(0)=v^{\prime \prime \prime}(0)=0, \\
& v^{\prime}(0)=v(1)=\beta \int_{0}^{1} v(s) d s,
\end{aligned}
$$

In this article, we first demonstrate a fixed point result under certain contraction in the setting of controlled $b$-Branciari metric type spaces.

- Thereafter, we specifically consider a following boundary value problem (BVP) for a singular fractional differential equation of order $\alpha$ :

where $3<\alpha<4,0<\beta<2,{ }^{C} D^{\alpha}$ is the Caputo fractional derivative and $h$ may be singular at $v=0$.

- Eventually, we investigate the existence and uniqueness of solutions of the aforementioned boundary value problem of order $\alpha$ via a fixed point problem of an integral operator.

MSC: $34 \mathrm{~B} 15 ; 54 \mathrm{H} 25$

Keywords: Controlled $b$-Branciari metric type spaces; Fixed point; Singular fractional differential equation; Integral boundary conditions; Green's function

\section{Springer}

\section{Introduction}

As regards science and engineering disciplines, fractional differential equations occur in the fields of aerodynamics, chemistry, physics, or polymer rheology electrodynamics, such as the mathematical simulation of structures and processes. The derivatives of fractional order $[28,35,38]$ are concerned in this sort of equations. Exceedingly, fractional-order differential equations often become tools for various perspectives on control systems, fluid dynamics, and so forth.

The significance of studying fractional-order differential equations arises from the factuality that fractional-order models are more precise than integer-order models; it seems

C) The Author(s) 2021. This article is licensed under a Creative Commons Attribution 4.0 International License, which permits use, sharing, adaptation, distribution and reproduction in any medium or format, as long as you give appropriate credit to the original author(s) and the source, provide a link to the Creative Commons licence, and indicate if changes were made. The images or other third party material in this article are included in the article's Creative Commons licence, unless indicated otherwise in a credit line to the material. If material is not included in the article's Creative Commons licence and your intended use is not permitted by statutory regulation or exceeds the permitted use, you will need to obtain permission directly from the copyright holder. To view a copy of this licence, visit http://creativecommons.org/licenses/by/4.0/. 
to be so that the fractional-order models have more degrees of freedom. Recent findings can be found in $[3,4,8,14,19,34,37,45]$ on fractional differential equations.

Integral boundary conditions have several applications in the areas such as problems with blood flow, thermo-elasticity, underground water supply, and population dynamics. We refer the reader to certain recent publications [11, 17, 22, 36, 42-44, 46, 48] and the references therein for a comprehensive explanation of the integral boundary conditions.

Many researchers have pointed out the importance of the existence and uniqueness of fractional differential equations of different orders [29, 30, 32, 35, 40]. The existence of positive solutions was considered especially in the context of cone metric spaces $[9,12,47]$.

On the other hand, fixed point theory can be used as a definitive modeling method in numerous fields and/or engineering to achieve solutions and/or research findings. In general, it has become one of the most effective features of modern mathematics and in particular of functional analysis. Fixed point theorems are concerned with the existence, uniqueness and characteristics of a specified operator's fixed points. The contraction mapping theorem due to Banach [10] is a very important and valuable finding on fixed point theory. Recent advancement in exploring new generalized metric spaces (and/or related results) has provoked great attention in metric fixed point theory (see $[1,5-7,13,16,18,20,23-$ $27,31,39])$.

\section{Preliminaries}

In the year 1993, Czerwik [15] initiated the idea of $b$-metric spaces as a generalization of metric spaces by multiplying a constant $b$ on the right side of the equation of triangle inequality.

Definition 2.1 Let $X \neq \emptyset$ and $b \geq 1$ be a given real number. A function $d_{b}: X \times X \rightarrow$ $[0,+\infty)$ is a $b$-metric if and only if for each $r, s, t \in X$ the following conditions are satisfied:

(1) $d_{b}(r, s) \geq 0$ for all $r, s \in X$ and $d_{b}(r, s)=0$ if and only if $r=s$;

(2) $d_{b}(r, s)=d_{b}(s, r)$ for all $r, s \in X$;

(3) $d_{b}(r, s) \leq b\left[d_{b}(r, t)+d_{b}(t, s)\right]$ for all $r, s, t \in X$.

Then $d_{b}$ is called a $b$-metric on $X$ and $\left(X, d_{b}\right)$ is called a $b$-metric space.

Nabil Mlaiki et al. [33] defined a new type of generalized $b$-metric spaces, namely controlled metric type spaces, as follows:

Definition 2.2 Let $X \neq \emptyset$ and $\omega: X \times X \rightarrow[1,+\infty)$. A function $d_{\omega}: X \times X \rightarrow[0,+\infty)$ is called a controlled metric type if:

(1) $d_{\omega}(r, s)=0$ if and only if $r=s$;

(2) $d_{\omega}(r, s)=d_{\omega}(s, r)$;

(3) $d_{\omega}(r, s) \leq \omega(r, t) d_{\omega}(r, t)+\omega(t, s) d_{\omega}(t, s)$

for all $r, s, t \in X$. The pair $\left(X, d_{\omega}\right)$ is called a controlled metric type space.

By concatenating the concepts of controlled metric type spaces [33] and extendedBranciari $b$-distance spaces [2], the authors in [41] proposed a new sort of metric spaces, namely controlled $b$-Branciari metric type spaces, that are defined now.

Definition 2.3 Let $X \neq \emptyset$ and $\mathfrak{C}: X \times X \rightarrow[1,+\infty)$. A function $d_{\mathfrak{C}}: X \times X \rightarrow[0,+\infty)$ is called a controlled $b$-Branciari metric type if it satisfies: 
(1) $d_{\mathfrak{C}}(r, s)=0$ if and only if $r=s$ for all $r, s \in X$;

(2) $d_{\mathfrak{C}}(r, s)=d_{\mathfrak{C}}(s, r)$ for all $r, s \in X$;

(3) $d_{\mathfrak{C}}(r, s) \leq \mathfrak{C}(r, t) d_{\mathfrak{C}}(r, t)+\mathfrak{C}(t, w) d_{\mathfrak{C}}(t, w)+\mathfrak{C}(w, s) d_{\mathfrak{C}}(w, s)$,

for all $r, s \in X$ and for all distinct points $t, w \in X$, each distinct from $r$ and $s$, respectively. The pair $\left(X, d_{\mathfrak{C}}\right)$ is named a controlled $b$-Branciari metric type space.

The major aspect of controlled $b$-Branciari metric type space is the extension of the rectangular inequality.

Example 2.4 ([41]) Let $X=\{1,2,3,4\}$. Define $d_{\mathfrak{C}}: X \times X \rightarrow[0,+\infty)$ as follows:

$$
\begin{aligned}
& d_{\mathfrak{C}}(r, r)=0, \forall r \in X, d_{\mathfrak{C}}(1, r)=d_{\mathfrak{C}}(r, 1)=50, \forall r \in X-\{1\}, \\
& d_{\mathfrak{C}}(2,3)=d_{\mathfrak{C}}(3,2)=d_{\mathfrak{C}}(2,4)=d_{\mathfrak{C}}(4,2)=200, \\
& d_{\mathfrak{C}}(4,3)=d_{\mathfrak{C}}(3,4)=800 .
\end{aligned}
$$

Let $\mathfrak{C}: X \times X \rightarrow[1,+\infty)$ be symmetric and can be defined as follows:

$$
\begin{aligned}
& \mathfrak{C}(r, r)=1, \forall r \in X, \\
& \mathfrak{C}(1,2)=3, \mathfrak{C}(1,3)=4, \mathfrak{C}(1,4)=\mathfrak{C}(2,3)=5, \mathfrak{C}(2,4)=6, \mathfrak{C}(3,4)=2 .
\end{aligned}
$$

Hence $\left(X, d_{\mathfrak{C}}\right)$ is a controlled $b$-Branciari metric type space. However, we can see that

(i) $d_{\mathfrak{C}}(3,4)=800>\mathfrak{C}(3,4)\left[d_{\mathfrak{C}}(3,1)+d_{\mathfrak{C}}(1,2)+d_{\mathfrak{C}}(2,4)\right]=600$,

(ii) $d_{\mathfrak{C}}(3,4)=800>\mathfrak{C}(3,1) d_{\mathfrak{C}}(3,1)+\mathfrak{C}(1,4) d_{\mathfrak{C}}(1,4)=450$.

Thus $\left(X, d_{\mathfrak{C}}\right)$ is neither an extended Branciari $b$-distance space nor a controlled metric type space.

For the reader's convenience, we present some necessary definitions and lemmas from the theory of fractional calculus.

Definition 2.5 ([21]) For a function $v:[0, \infty) \rightarrow R$, the Caputo derivative of fractional order $\alpha>0, n-1<\alpha<n, n \in \mathbb{N}$ is defined as

$$
{ }^{c} D^{\alpha} v(t)=\frac{1}{\Gamma(n-\alpha)} \int_{0}^{t} \frac{v^{n}(s)}{(t-s)^{\alpha-n+1}} d s, \quad n=[\alpha]+1,
$$

where $[\alpha]$ denotes the integer part of the real number $\alpha$.

Definition 2.6 ([21]) The Riemann-Liouville fractional integral of order $\alpha$ for a function $v$ is defined as

$$
I^{\alpha} v(t)=\frac{1}{\Gamma(\alpha)} \int_{0}^{t}(t-s)^{\alpha-1} v(s) d s, \quad \alpha>0
$$

provided that such an integral exists.

Lemma 2.7 [21] Given $y \in C(0,1) \cup L(0,1), 3<\alpha<4$ and $0<\beta<2$, the unique solution of

$$
\begin{aligned}
& { }^{c} D^{\alpha} v(t)+y(t)=0, \quad 0<t<1, \\
& v^{\prime \prime}(0)=v^{\prime \prime \prime}(0)=0, \\
& v^{\prime}(0)=v(1)=\beta \int_{0}^{1} v(s) d s,
\end{aligned}
$$




$$
v(t)=\int_{0}^{1} G(t, s) y(s) d s
$$

where

$$
G(t, s)=\frac{1}{\alpha(2-\beta) \Gamma(\alpha)} \begin{cases}\{\alpha(2-\beta)+2 \beta t(\alpha-1+s)\}(1-s)^{\alpha-1} & \\ -\alpha(2-\beta)(t-s)^{\alpha-1}, & \text { if } 0 \leq s \leq t \leq 1 \\ \{\alpha(2-\beta)+2 \beta t(\alpha-1+s)\}(1-s)^{\alpha-1}, & \text { if } 0 \leq t \leq s \leq 1\end{cases}
$$

Lemma 2.8 ([28]) If $\alpha>0$ and $\beta>0$, then

1. ${ }^{c} D^{\alpha} t^{\beta-1}=\frac{\Gamma(\beta)}{\Gamma(\beta-\alpha)} t^{\beta-\alpha-1}$, for $\beta>n$.

2. ${ }^{c} D^{\alpha} t^{k}=0$, for $k=0,1,2, \ldots, n-1$.

Deeply influenced by the foregoing facts, we specifically discuss the following boundary value problem (BVP) for a singular fractional differential equation of order $\alpha$ :

$$
\begin{aligned}
& { }^{c} D^{\alpha} v(t)+h(t, v(t))=0, \quad 0<t<1, \\
& v^{\prime \prime}(0)=v^{\prime \prime \prime}(0)=0, \\
& v^{\prime}(0)=v(1)=\beta \int_{0}^{1} v(s) d s,
\end{aligned}
$$

where $3<\alpha<4,0<\beta<2,{ }^{c} D^{\alpha}$ is the Caputo fractional derivative given by (2.1) and $h$ may be singular at $v=0$.

Ying He [21] takes into consideration the problem (2.4) for a continuous function $h$ and $\beta \in(0, \alpha)$. The outcomes in the paper correspond to the positive solutions to this problem. Ying He [21] first developed the accurate estimation of the below BVP of the Green's function, and unveiled some of its properties;

$$
\begin{aligned}
& { }^{c} D^{\alpha} v(t)+y(t)=0, \quad 0<t<1, \\
& v^{\prime \prime}(0)=v^{\prime \prime \prime}(0)=0, \\
& v^{\prime}(0)=v(1)=\beta \int_{0}^{1} v(s) d s .
\end{aligned}
$$

- In Sect. 3, we prove a fixed point theorem in the framework of controlled $b$-Branciari metric type spaces.

- In Sect. 4, an approximation for the Green's function relevant to the problem is presented and utilized for the solution of the given problem in the proof of the existence and uniqueness theorem.

- Thereafter the BVP given in Eq. (2.4) is transformed into an integral equation and analyzed as a fixed point problem.

- Criteria for the existence and uniqueness of a fixed point for an integral operator are evaluated via complete controlled $b$-Branciari metric type spaces. Eventually in Sect. 5, an exemplary example is offered to endorse the theoretical result. 


\section{Fixed point theorem}

Throughout this section, in the sense of controlled $b$-Branciari metric type spaces, we give a fixed point result under specific contraction condition.

Theorem 3.1 Let $\left(X, d_{\mathfrak{C}}\right)$ be a complete controlled b-Branciari metric type space with coefficient function $\mathfrak{C}(r, s)>1$ for any $r, s \in X$ and $F: X \rightarrow X$ be a mapping satisfying

$$
d_{\mathfrak{C}}(F r, F s) \leq \eta \mathfrak{C}(r, s) d_{\mathfrak{C}}(r, s), \quad \text { for all } r, s \in X,
$$

where $\eta \in[0,1)$ is such that, for any $r_{0} \in X$, we have

$$
\lim \sup _{n, m \rightarrow+\infty} \mathfrak{C}\left(r_{n}, r_{m}\right) \mathfrak{C}\left(r_{n}, r_{n+1}\right)<\frac{1}{\eta^{2}}
$$

and

$$
\lim \sup _{n, m \rightarrow+\infty} \mathfrak{C}\left(r_{n}, r_{m}\right) \mathfrak{C}\left(r_{n+1}, r_{n+2}\right)<\frac{1}{\eta^{2}}
$$

and we assume $\lim \sup _{n \rightarrow+\infty} \mathfrak{C}\left(r, r_{n}\right)$ and $\limsup _{n \rightarrow+\infty} \mathfrak{C}\left(r_{n}, r\right)$ exist for any $r \in X$. Then $F$ has a fixed point in $X$. Moreover, suppose that, for any $r, s \in X$, we have

$$
\lim \sup _{n \rightarrow+\infty} \mathfrak{C}\left(F^{n} r, F^{n} s\right)<\frac{1}{\eta}
$$

where $F^{n} r=F^{n-1}(F r)$. Then the fixed point of $F$ is unique.

Proof Let $r_{0} \in X$ and define an iterative sequence $\left\{r_{n}\right\}$ by

$$
r_{0}, \quad F r_{0}=r_{1}, \quad F r_{1}=r_{2} \Rightarrow r_{2}=F^{2} r_{0}, \ldots, r_{n+1}=F^{n+1} r_{0} .
$$

Consider

$$
\begin{aligned}
d_{\mathfrak{C}}\left(r_{n}, r_{n+1}\right) & =d_{\mathfrak{C}}\left(F r_{n-1}, F r_{n}\right) \\
& \leq \eta \mathfrak{C}\left(r_{n-1}, r_{n}\right) d_{\mathfrak{C}}\left(r_{n-1}, r_{n}\right) \\
& \vdots \\
& \leq \eta^{n} \prod_{k=1}^{n} \mathfrak{C}\left(r_{k-1}, r_{k}\right) d_{\mathfrak{C}}\left(r_{0}, r_{1}\right) .
\end{aligned}
$$

Similarly

$$
\begin{aligned}
d_{\mathfrak{C}}\left(r_{n}, r_{n+2}\right) & =d_{\mathfrak{C}}\left(F r_{n-1}, F r_{n+1}\right) \\
& \leq \eta \mathfrak{C}\left(r_{n-1}, r_{n+1}\right) d_{\mathfrak{C}}\left(r_{n-1}, r_{n+1}\right) \\
& \vdots \\
& \leq \eta^{n} \prod_{k=1}^{n} \mathfrak{C}\left(r_{k-1}, r_{k+1}\right) d_{\mathfrak{C}}\left(r_{0}, r_{2}\right) .
\end{aligned}
$$

Now to show that $\left\{r_{n}\right\}$ is Cauchy, we consider $d_{\mathfrak{C}}\left(r_{n}, r_{n+p}\right)$ in two cases. 
Case 1: If $p$ is odd, say $2 m+1$, then using (3.5), we obtain

$$
\begin{aligned}
& d_{\mathfrak{C}}\left(r_{n}, r_{n+2 m+1}\right) \leq \mathfrak{C}\left(r_{n}, r_{n+1}\right) d_{\mathfrak{C}}\left(r_{n}, r_{n+1}\right)+\mathfrak{C}\left(r_{n+1}, r_{n+2}\right) d_{\mathfrak{C}}\left(r_{n+1}, r_{n+2}\right) \\
& +\mathfrak{C}\left(r_{n+2}, r_{n+2 m+1}\right) d_{\mathfrak{C}}\left(r_{n+2}, r_{n+2 m+1}\right) \\
& \vdots \\
& \leq \mathfrak{C}\left(r_{n}, r_{n+1}\right) d_{\mathfrak{C}}\left(r_{n}, r_{n+1}\right)+\mathfrak{C}\left(r_{n+1}, r_{n+2}\right) d_{\mathfrak{C}}\left(r_{n+1}, r_{n+2}\right) \\
& +\sum_{i=\frac{n}{2}+1}^{\frac{n+2 m-2}{2}}\left[\mathfrak{C}\left(r_{2 i}, r_{2 i+1}\right) d_{\mathfrak{C}}\left(r_{2 i}, r_{2 i+1}\right)+\mathfrak{C}\left(r_{2 i+1}, r_{2 i+2}\right) d_{\mathfrak{C}}\left(r_{2 i+1}, r_{2 i+2}\right)\right] \\
& \times \prod_{\mathfrak{j}=\frac{n}{2}+1}^{i} \mathfrak{C}\left(r_{2 \mathrm{j}}, r_{n+2 m+1}\right)+\prod_{i=\frac{n}{2}+1}^{\frac{n+2 m}{2}} \mathfrak{C}\left(r_{2 i}, r_{n+2 m+1}\right) d_{\mathfrak{C}}\left(r_{n+2 m}, r_{n+2 m+1}\right) \\
& \leq \sum_{i=\frac{n}{2}}^{\frac{n+2 m}{2}} \mathfrak{C}\left(r_{2 i}, r_{2 i+1}\right) d_{\mathfrak{C}}\left(r_{2 i}, r_{2 i+1}\right) \prod_{\mathfrak{j}=\frac{n}{2}}^{i} \mathfrak{C}\left(r_{2 j}, r_{n+2 m+1}\right) \\
& \left.+\sum_{i=\frac{n}{2}}^{\frac{n+2 m-2}{2}} \mathfrak{C}\left(r_{2 i+1}, r_{2 i+2}\right) d_{\mathfrak{C}}\left(r_{2 i+1}, r_{2 i+2}\right)\right] \prod_{\mathfrak{j}=\frac{n}{2}}^{i} \mathfrak{C}\left(r_{2 j}, r_{n+2 m+1}\right) \\
& \leq \sum_{i=\frac{n}{2}}^{\frac{n+2 m}{2}} \mathfrak{C}\left(r_{2 i}, r_{2 i+1}\right) \prod_{j=\frac{n}{2}}^{i} \mathfrak{C}\left(r_{2 j}, r_{n+2 m+1}\right) \eta^{2 i} \prod_{k=1}^{2 i} \mathfrak{C}\left(r_{k-1}, r_{k}\right) d_{\mathfrak{C}}\left(r_{0}, r_{1}\right) \\
& +\sum_{i=\frac{n}{2}}^{\frac{n+2 m-2}{2}} \mathfrak{C}\left(r_{2 i+1}, r_{2 i+2}\right) \prod_{\mathfrak{j}=\frac{n}{2}}^{i} \mathfrak{C}\left(r_{2 \mathfrak{j}}, r_{n+2 m+1}\right) \eta^{2 i+1} \prod_{k=1}^{2 i+1} \mathfrak{C}\left(r_{k-1}, r_{k}\right) d_{\mathfrak{C}}\left(r_{0}, r_{1}\right)
\end{aligned}
$$

Let

$$
a_{i}=\eta^{2 i} \prod_{j=\frac{n}{2}}^{i} \mathfrak{C}\left(r_{2 j}, r_{n+2 m+1}\right) \prod_{k=1}^{2 i+1} \mathfrak{C}\left(r_{k-1}, r_{k}\right) d_{\mathfrak{C}}\left(r_{0}, r_{1}\right)
$$

and

$$
b_{i}=\eta^{2 i+1} \prod_{j=\frac{n}{2}}^{i} \mathfrak{C}\left(r_{2 j}, r_{n+2 m+1}\right) \prod_{k=1}^{2 i+2} \mathfrak{C}\left(r_{k-1}, r_{k}\right) d_{\mathfrak{C}}\left(r_{0}, r_{1}\right)
$$

By utilizing (3.2) and (3.3), we obtain

$$
\lim \sup _{i \rightarrow+\infty} \frac{a_{i+1}}{a_{i}}=\lim \sup _{i, m \rightarrow+\infty} \eta^{2} \mathfrak{C}\left(r_{2 i+2}, r_{n+2 m+1}\right) \mathfrak{C}\left(r_{2 i+2}, r_{2 i+3}\right)<1
$$

and

$\lim \sup _{i \rightarrow+\infty} \frac{b_{i+1}}{b_{i}}=\lim \sup _{i, m \rightarrow+\infty} \eta^{2} \mathfrak{C}\left(r_{2 i+2}, r_{n+2 m+1}\right) \mathfrak{C}\left(r_{2 i+3}, r_{2 i+4}\right)<1$ 
Thereby we deduce that

$$
\sum_{i=\frac{n}{2}}^{+\infty} \eta^{2 i} \prod_{j=\frac{n}{2}}^{i} \mathfrak{C}\left(r_{2 j}, r_{n+2 m+1}\right) \prod_{k=1}^{2 i+1} \mathfrak{C}\left(r_{k-1}, r_{k}\right) d_{\mathfrak{C}}\left(r_{0}, r_{1}\right)<+\infty
$$

and

$$
\sum_{i=\frac{n}{2}}^{+\infty} \eta^{2 i+1} \prod_{j=\frac{n}{2}}^{i} \mathfrak{C}\left(r_{2 j}, r_{n+2 m+1}\right) \prod_{k=1}^{2 i+2} \mathfrak{C}\left(r_{k-1}, r_{k}\right) d_{\mathfrak{C}}\left(r_{0}, r_{1}\right)<+\infty
$$

Henceforth

$$
\left(\sum_{i=\frac{n}{2}}^{\frac{n+2 m}{2}} \eta^{2 i} \prod_{j=\frac{n}{2}}^{i+1} \mathfrak{C}\left(r_{2 j}, r_{n+2 m+1}\right) \prod_{k=1}^{2 i+1} \mathfrak{C}\left(r_{k-1}, r_{k}\right) d_{\mathfrak{C}}\left(r_{0}, r_{1}\right)\right)
$$

and

$$
\left(\sum_{i=\frac{n}{2}}^{\frac{n+2 m-2}{2}} \eta^{2 i+1} \prod_{j=\frac{n}{2}}^{i} \mathfrak{C}\left(r_{2 j}, r_{n+2 m+1}\right) \prod_{k=1}^{2 i+2} \mathfrak{C}\left(r_{k-1}, r_{k}\right) d_{\mathfrak{C}}\left(r_{0}, r_{1}\right)\right)
$$

are Cauchy sequences in $\mathbb{R}$.

Case 2: If $p$ is even, say $2 m$, using (3.5) and (3.6), we obtain

$$
\begin{aligned}
& d_{\mathfrak{C}}\left(r_{n}, r_{n+2 m}\right) \leq \mathfrak{C}\left(r_{n}, r_{n+2}\right) d_{\mathfrak{C}}\left(r_{n}, r_{n+2}\right)+\mathfrak{C}\left(r_{n+2}, r_{n+3}\right) d_{\mathfrak{C}}\left(r_{n+2}, r_{n+3}\right) \\
& +\mathfrak{C}\left(r_{n+3}, r_{n+2 m}\right) d_{\mathfrak{C}}\left(r_{n+3}, r_{n+2 m}\right) \\
& \leq \mathfrak{C}\left(r_{n}, r_{n+2}\right) d_{\mathfrak{C}}\left(r_{n}, r_{n+2}\right)+\mathfrak{C}\left(r_{n+2}, r_{n+3}\right) d_{\mathfrak{C}}\left(r_{n+2}, r_{n+3}\right) \\
& +\sum_{i=\frac{n+3}{2}}^{\frac{n+2 m-3}{2}}\left[\mathfrak{C}\left(r_{2 i}, r_{2 i+1}\right) d_{\mathfrak{C}}\left(r_{2 i}, r_{2 i+1}\right)+\mathfrak{C}\left(r_{2 i+1}, r_{2 i+2}\right) d_{\mathfrak{C}}\left(r_{2 i+1}, r_{2 i+2}\right)\right] \\
& \times \prod_{\mathfrak{j}=\frac{n+3}{2}}^{i} \mathfrak{C}\left(r_{2 \mathrm{j}}, r_{n+2 m}\right)+\prod_{i=\frac{n+3}{2}}^{\frac{n+2 m-1}{2}} \mathfrak{C}\left(r_{2 i}, r_{n+2 m}\right) d_{\mathfrak{C}}\left(r_{n+2 m-1}, r_{n+2 m}\right) \\
& \leq \mathfrak{C}\left(r_{n}, r_{n+2}\right) d_{\mathfrak{C}}\left(r_{n}, r_{n+2}\right) \\
& +\sum_{i=\frac{n+3}{2}}^{\frac{n+2 m-1}{2}} \mathfrak{C}\left(r_{2 i}, r_{2 i+1}\right) d_{\mathfrak{C}}\left(r_{2 i}, r_{2 i+1}\right) \prod_{\mathfrak{j}=\frac{n+3}{2}}^{i} \mathfrak{C}\left(r_{2 \mathfrak{j}}, r_{n+2 m}\right) \\
& \left.+\sum_{i=\frac{n+1}{2}}^{\frac{n+2 m-3}{2}} \mathfrak{C}\left(r_{2 i+1}, r_{2 i+2}\right) d_{\mathfrak{C}}\left(r_{2 i+1}, r_{2 i+2}\right)\right] \prod_{j=\frac{n+1}{2}}^{i} \mathfrak{C}\left(r_{2 j}, r_{n+2 m}\right) \\
& \leq \mathfrak{C}\left(r_{n}, r_{n+2}\right) \eta^{n} \prod_{i=1}^{n} \mathfrak{C}\left(r_{i-1}, r_{i+1}\right) d_{\mathfrak{C}}\left(r_{0}, r_{2}\right)
\end{aligned}
$$




$$
\begin{aligned}
& +\sum_{i=\frac{n+3}{2}}^{\frac{n+2 m-1}{2}} \mathfrak{C}\left(r_{2 i}, r_{2 i+1}\right) \prod_{\mathfrak{j}=\frac{n+3}{2}}^{i} \mathfrak{C}\left(r_{2 \mathfrak{j}}, r_{n+2 m}\right) \eta^{2 i} \prod_{i=1}^{2 i} \mathfrak{C}\left(r_{k-1}, r_{k}\right) d_{\mathfrak{C}}\left(r_{0}, r_{1}\right) \\
& +\sum_{i=\frac{n+1}{2}}^{\frac{n+2 m-3}{2}} \mathfrak{C}\left(r_{2 i+1}, r_{2 i+2}\right) \prod_{\mathfrak{j}=\frac{n+1}{2}}^{i} \mathfrak{C}\left(r_{2 \mathfrak{j}}, r_{n+2 m}\right) \eta^{2 i+1} \prod_{i=1}^{2 i+1} \mathfrak{C}\left(r_{k-1}, r_{k}\right) d_{\mathfrak{C}}\left(r_{0}, r_{1}\right)
\end{aligned}
$$

Let

$$
a_{i}=\eta^{2 i} \prod_{j=\frac{n+3}{2}}^{i} \mathfrak{C}\left(r_{2 j}, r_{n+2 m}\right) \prod_{k=1}^{2 i+1} \mathfrak{C}\left(r_{k-1}, r_{k}\right) d_{\mathfrak{C}}\left(r_{0}, r_{1}\right)
$$

and

$$
b_{i}=\eta^{2 i+1} \prod_{j=\frac{n+1}{2}}^{i} \mathfrak{C}\left(r_{2 j}, r_{n+2 m}\right) \prod_{k=1}^{2 i+2} \mathfrak{C}\left(r_{k-1}, r_{k}\right) d_{\mathfrak{C}}\left(r_{0}, r_{1}\right)
$$

By using (3.2) and (3.3), we get

$$
\lim \sup _{i \rightarrow+\infty} \frac{a_{i+1}}{a_{i}}=\lim \sup _{i, m \rightarrow+\infty} \eta^{2} \mathfrak{C}\left(r_{2 i+2}, r_{n+2 m}\right) \mathfrak{C}\left(r_{2 i+2}, r_{2 i+3}\right)<1
$$

and

$$
\lim \sup _{i \rightarrow+\infty} \frac{b_{i+1}}{b_{i}}=\lim \sup _{i, m \rightarrow+\infty} \eta^{2} \mathfrak{C}\left(r_{2 i+2}, r_{n+2 m}\right) \mathfrak{C}\left(r_{2 i+3}, r_{2 i+4}\right)<1
$$

Hence, we deduce that

$$
\sum_{i=\frac{n+3}{2}}^{+\infty} \eta^{2 i} \prod_{j=\frac{n+3}{2}}^{i} \mathfrak{C}\left(r_{2 j}, r_{n+2 m}\right) \prod_{k=1}^{2 i+1} \mathfrak{C}\left(r_{k-1}, r_{k}\right) d_{\mathfrak{C}}\left(r_{0}, r_{1}\right)<+\infty
$$

and

$$
\sum_{i=\frac{n+1}{2}}^{+\infty} \eta^{2 i+1} \prod_{j=\frac{n+1}{2}}^{i} \mathfrak{C}\left(r_{2 j}, r_{n+2 m}\right) \prod_{k=1}^{2 i+2} \mathfrak{C}\left(r_{k-1}, r_{k}\right) d_{\mathfrak{C}}\left(r_{0}, r_{1}\right)<+\infty
$$

Thereby

$$
\left(\sum_{i=\frac{n+3}{2}}^{\frac{n+2 m-1}{2}} \eta^{2 i} \prod_{j=\frac{n+3}{2}}^{i} \mathfrak{C}\left(r_{2 j}, r_{n+2 m+1}\right) \prod_{k=1}^{2 i+1} \mathfrak{C}\left(r_{k-1}, r_{k}\right) d_{\mathfrak{C}}\left(r_{0}, r_{1}\right)\right)
$$

and

$$
\left(\sum_{i=\frac{n+1}{2}}^{\frac{n+2 m-3}{2}} \eta^{2 i+1} \prod_{j=\frac{n+1}{2}}^{i} \mathfrak{C}\left(r_{2 j}, r_{n+2 m+1}\right) \prod_{k=1}^{2 i+2} \mathfrak{C}\left(r_{k-1}, r_{k}\right) d_{\mathfrak{C}}\left(r_{0}, r_{1}\right)\right)
$$


are Cauchy sequences in $\mathbb{R}$. By (3.7) and (3.8), it implies that $\left\{r_{n}\right\}$ is a Cauchy sequence in $X$ when $n \rightarrow+\infty$. Through completeness of $\left(X, d_{\mathfrak{C}}\right)$ there exists $r \in X$ such that $\lim _{n \rightarrow+\infty} r_{n}=$ $r$. We are now attempting to demonstrate that $r$ is a fixed point of $F$. For $n \in \mathbb{N}$, we have

$$
d_{\mathfrak{C}}\left(r_{n+2}, r\right) \leq \mathfrak{C}\left(r_{n+2}, r_{n+1}\right) d_{\mathfrak{C}}\left(r_{n+2}, r_{n+1}\right)+\mathfrak{C}\left(r_{n+1}, r_{n}\right) d_{\mathfrak{C}}\left(r_{n+1}, r_{n}\right)+\mathfrak{C}\left(r_{n}, r\right) d_{\mathfrak{C}}\left(r_{n}, r\right) .
$$

Passing $n \rightarrow+\infty$ in the aforementioned inequality, we find

$$
\begin{aligned}
\lim _{n \rightarrow+\infty} d_{\mathfrak{C}} & \left(r_{n+2}, r\right)=0, \\
d_{\mathfrak{C}}(r, F r) \leq & \mathfrak{C}\left(r, r_{n+2}\right) d_{\mathfrak{C}}\left(r, r_{n+2}\right)+\mathfrak{C}\left(r_{n+2}, r_{n+1}\right) d_{\mathfrak{C}}\left(r_{n+2}, r_{n+1}\right)+\mathfrak{C}\left(r_{n+1}, F r\right) d_{\mathfrak{C}}\left(r_{n+1}, F r\right) \\
= & \mathfrak{C}\left(r, r_{n+2}\right) d_{\mathfrak{C}}\left(r, r_{n+2}\right)+\mathfrak{C}\left(r_{n+2}, r_{n+1}\right) d_{\mathfrak{C}}\left(r_{n+2}, r_{n+1}\right)+\mathfrak{C}\left(r_{n+1}, F r\right) d_{\mathfrak{C}}\left(F r_{n}, F r\right) \\
\leq & \mathfrak{C}\left(r, r_{n+2}\right) d_{\mathfrak{C}}\left(r, r_{n+2}\right)+\mathfrak{C}\left(r_{n+2}, r_{n+1}\right) d_{\mathfrak{C}}\left(r_{n+2}, r_{n+1}\right) \\
& +\mathfrak{C}\left(r_{n+1}, F r\right) \eta \mathfrak{C}\left(r_{n}, r\right) d_{\mathfrak{C}}\left(r_{n}, r\right) .
\end{aligned}
$$

By employing Eq. (3.9) and by the hypothesis of the theorem, we get $d_{\mathfrak{C}}(F r, r) \leq 0$ as $n \rightarrow$ $+\infty$. Therefore $d_{\mathfrak{C}}(r, F r)=0$ i.e., $F r=r$. As a result we see that $r$ is a fixed point of $F$.

Unicity:Let $r, s$ be the two fixed points of $F$ where $r \neq s$, then $F r=r$ and $F s=s$. Consider

$$
d_{\mathfrak{C}}(r, s)=d_{\mathfrak{C}}(F r, F s) \leq \eta \mathfrak{C}(r, s) d_{\mathfrak{C}}(r, s)=\eta \mathfrak{C}\left(F^{n} r, F^{n} s\right) d_{\mathfrak{C}}(r, s) .
$$

Letting $n \rightarrow+\infty$ in the equation above and utilizing (3.4), we obtain $d_{\mathfrak{C}}(r, s)<d_{\mathfrak{C}}(r, s)$, which is a contradiction. Thereby, $r$ is a unique fixed point of $F$.

Corollary 3.2 Let $\left(X, d_{r}\right)$ be a complete rectangular $b$-metric space with $b \geq 1$. Let $F: X \rightarrow$ $X$ be a mapping. Assume there exists $l \in[0,1)$ such that

$$
d_{r}(F r, F s) \leq b l d_{r}(r, s)
$$

for all $r, s \in X$. Assume that $b^{2} l<1$. Then $F$ has a unique fixed point $r^{*}$.

Proof The proof follows from Theorem 3.1 by defining $\mathfrak{C}: X \times X \rightarrow[1,+\infty)$ as $\mathfrak{C}(r, s)=$ $b$.

\section{Existence-uniqueness of the solution of the BVP (2.4)}

In this section, we confirm the existence and uniqueness of the solution of the nonlinear BVP (2.4) within controlled $b$-Branciari metric type spaces.

We commence this section by proposing the Green's function developed in Ying He [21] relevant to the BVP corresponding to the linear fractional equation (2.5). Thereafter we present an inequality fulfilled by the Green's function to be utilized on the nonlinear BVP (2.4) in our existence-unicity result. It is proved in Ying He [21] that linear problem (2.5) has a unique solution in $C[0,1]$ given by

$$
v(t)=\int_{0}^{1} G(t, s) y(s) d s
$$


where $G(t, s)$ is the Green's function defined by

$$
G(t, s)=\frac{1}{\alpha(2-\beta) \Gamma(\alpha)} \begin{cases}\{\alpha(2-\beta)+2 \beta t(\alpha-1+s)\}(1-s)^{\alpha-1} & \\ -\alpha(2-\beta)(t-s)^{\alpha-1}, & \text { if } 0 \leq s \leq t \leq 1, \\ \{\alpha(2-\beta)+2 \beta t(\alpha-1+s)\}(1-s)^{\alpha-1}, & \text { if } 0 \leq t \leq s \leq 1\end{cases}
$$

where $3<\alpha<4$ and $0<\beta<2$. For the properties of the Green's function, we refer to Ying He [21].

In the following, we provide an estimation for the $L^{2}$ norm of the Green's function of a fractional differential equation with integral boundary conditions specified in (2.4).

Lemma 4.1 Let $3<\alpha<4$ and $0<\beta<2, \alpha \neq \beta$ hold. Then, for all $t, s \in(0,1)$, the Green's function $G(t, \cdot) \in L_{2}$ obeys

$$
\int_{0}^{1}|G(t, s)|^{2} d s<\frac{1}{(\Gamma(\alpha))^{2}}\left[\frac{4}{5}+\frac{12 \beta}{5|\beta-2|}+\frac{20 \beta^{2}}{21(\beta-2)^{2}}\right] .
$$

Proof If $3<\alpha<4$ and $0<\beta<2, \beta \neq \alpha$, the interpretation of the Green's function specifies:

(1) For $0 \leq s \leq t \leq 1$,

$$
\begin{aligned}
|G(t, s)| & \leq \frac{2 \alpha|\beta-2|+2 \beta t(\alpha-1+s)}{\alpha|\beta-2| \Gamma(\alpha)}(1-s)^{\alpha-1} \\
& \leq \frac{1}{\Gamma(\alpha)}\left(2+\frac{2 \beta}{\alpha|\beta-2|}(\alpha-1+s)\right)(1-s)^{\alpha-1} .
\end{aligned}
$$

(2) For $0 \leq t \leq s \leq 1$,

$$
\begin{aligned}
|G(t, s)| & \leq \frac{\alpha|\beta-2|+2 \beta t(\alpha-1+s)}{\alpha|\beta-2| \Gamma(\alpha)}(1-s)^{\alpha-1} \\
& \leq \frac{1}{\Gamma(\alpha)}\left(1+\frac{2 \beta}{\alpha|\beta-2|}(\alpha-1+s)\right)(1-s)^{\alpha-1}
\end{aligned}
$$

Therefore, by Eqs. (4.3) and (4.4), for all $t, s \in(0,1)$, we have

$$
|G(t, s)|^{2} \leq \frac{1}{(\Gamma(\alpha))^{2}}\left(4+\frac{8 \beta(\alpha-1+s)}{\alpha|\beta-2|}+\frac{4 \beta^{2}(\alpha-1+s)^{2}}{\alpha^{2}(\beta-2)^{2}}\right)(1-s)^{2 \alpha-2},
$$

which yields

$$
\begin{aligned}
\int_{0}^{1}|G(t, s)|^{2} d s \\
\leq \frac{1}{(\Gamma(\alpha))^{2}}\left[\int_{0}^{1} 4(1-s)^{2 \alpha-2} d s+\frac{8 \beta}{\alpha|\beta-2|} \int_{0}^{1}(\alpha-1+s)(1-s)^{2 \alpha-2} d s\right. \\
\left.\quad+\frac{4 \beta^{2}}{\alpha^{2}(\beta-2)^{2}} \int_{0}^{1}(\alpha-1+s)^{2}(1-s)^{2 \alpha-2} d s\right] \\
=\frac{1}{(\Gamma(\alpha))^{2}}\left[\frac{4}{2 \alpha-1}+\frac{8 \beta}{\alpha|\beta-2|}\left(\frac{2 \alpha^{2}-2 \alpha+1}{2 \alpha(2 \alpha-1)}\right)+\frac{4 \beta^{2}}{\alpha(\beta-2)^{2}}\left(\frac{2 \alpha^{2}-3 \alpha+2}{(2 \alpha-1)(2 \alpha+1)}\right)\right] \\
<\frac{1}{(\Gamma(\alpha))^{2}}\left[\frac{4}{5}+\frac{12 \beta}{5|\beta-2|}+\frac{20 \beta^{2}}{21(\beta-2)^{2}}\right] .
\end{aligned}
$$


Let $h(\cdot, v(\cdot)) \in L_{2}$ for any $v \in C[0,1]$ and define a mapping $F: C[0,1] \rightarrow C[0,1]$ as

$$
F v(t)=\int_{0}^{1} G(t, s) h(s, v(s)) d s, \quad t \in[0,1]
$$

where $s \rightarrow G(t, s)$ is continuous from $[0,1] \rightarrow L_{2}$. Let $t_{n} \in[0,1]$ with $t_{n} \rightarrow t$.

As $h(\cdot, v(\cdot)), G(t, \cdot) \in L_{2}$, for any $v \in C[0,1]$ and $t \in[0,1]$, the function $G(t, \cdot) h(\cdot, v(\cdot))$ is integrable. Then, by the Lebesgue dominated convergence theorem, we have

$$
\begin{aligned}
\lim _{n \rightarrow \infty} F v\left(t_{n}\right) & =\lim _{n \rightarrow \infty} \int_{0}^{1} G\left(t_{n}, s\right) h(s, v(s)) d s \\
& =\int_{0}^{1} \lim _{n \rightarrow \infty} G\left(t_{n}, s\right) h(s, v(s)) d s \\
& =\int_{0}^{1} G(t, s) h(s, v(s)) d s=F v(t),
\end{aligned}
$$

which implies $F v \in C[0,1]$. Hence the map $F: C[0,1] \rightarrow C[0,1]$ holds.

Let $d_{\mathfrak{C}}: C[0,1] \times C[0,1] \rightarrow[0,+\infty)$ be defined by

$$
d_{\mathfrak{C}}(v, z)=\sup _{t \in[0,1]}|v(t)-z(t)|^{2}
$$

Then $d_{\mathfrak{C}}$ is a complete controlled $b$-Branciari metric type on $C[0,1]$ with a controlled function

$$
\mathfrak{C}(v, z)= \begin{cases}9+\sup _{t \in[0,1]}|v(t)-z(t)|^{2}, & \text { if } v(t) \neq z(t) \\ 1, & \text { if } v(t)=z(t)\end{cases}
$$

Lemma 4.2 Let $v \in C[0,1]$ and $F$ be given by Eq. (4.6). Then $v(t)$ is a solution of boundary value problem (2.4) if and only if it is the fixed point of $F$.

Proof Let $v(t)$ be a solution of the BVP (2.4). Then, by Lemma 2.7, the unique solution of (2.4) can be represented as

$$
v(t)=\int_{0}^{1} G(t, s) h(s, v(s)) d s, \quad t \in[0,1]
$$

where $G(t, s)$ is defined in (2.3). Thereby $v(t)$ is a fixed point of $F$.

On the other hand, let $v(t)$ be a fixed point of $F$. As $\beta<n-1$, by Lemma 2.8, we have

$$
\begin{aligned}
{ }^{c} D^{\alpha}(v(t))= & { }^{c} D^{\alpha}\left(\int_{0}^{1} G(t, s) h(s, v(s)) d s\right) \\
= & \frac{1}{\alpha(2-\beta) \Gamma(\alpha)}\left[{ } ^ { c } D ^ { \alpha } \left(\int _ { 0 } ^ { t } \left[-\alpha(2-\beta)(t-s)^{\alpha-1}\right.\right.\right. \\
& \left.+[\alpha(2-\beta)+2 \beta t(\alpha-1+s)](1-s)^{\alpha-1}\right] h(s, v(s)) d s \\
& \left.\left.+\int_{t}^{1}[\alpha(2-\beta)+2 \beta t(\alpha-1+s)](1-s)^{\alpha-1} h(s, v(s)) d s\right)\right]
\end{aligned}
$$




$$
\begin{aligned}
= & -h(t, v(t))+{ }^{c} D^{\alpha}\left(\frac{t^{0}}{\Gamma(\alpha)} \int_{0}^{1}(1-s)^{\alpha-1} h(s, v(s)) d s\right. \\
& \left.+\frac{2 \beta t}{\alpha(2-\beta) \Gamma(\alpha)}(\alpha-1+s)(1-s)^{\alpha-1} h(s, v(s)) d s\right) \\
= & -h(t, v(t)) .
\end{aligned}
$$

Therefore $v(t)$ satisfies the differential equation (2.4). Moreover it is easy to verify that $v^{\prime \prime}(0)=v^{\prime \prime \prime}(0)=0$ and $v^{\prime}(0)=v(1)=\beta \int_{0}^{1} v(s) d s$, which implies $v(t)$ is a solution for the BVP (2.4). Since $v(t)$ is a fixed point of $F$ and $F$ is continuous, $v(t)$ is a continuous solution for the given boundary value problem. Hence, every fixed point of $F$ in $C[0,1]$ solves the BVP (2.4).

We propose the following existence-uniqueness theorem for the solution of the problem (2.4).

Theorem 4.3 Let $3<\alpha<4$ and

$$
\eta=\frac{1}{(\Gamma(\alpha))^{2}}\left[\frac{4}{5}+\frac{12 \beta}{5|\beta-2|}+\frac{20 \beta^{2}}{21(\beta-2)^{2}}\right]<1
$$

hold for any $0<\beta<2, \beta \neq \alpha$. Suppose that for the function $h(\cdot, v(\cdot)) \in L_{2}$ for any $v \in C[0,1]$ and for any $v, z \in C[0,1]$, the inequality

$$
|h(s, v(s))-h(s, z(s))|^{2} \leq\left(9+|v(s)-z(s)|^{2}\right)|v(s)-z(s)|^{2}, \quad s \in[0,1]
$$

holds. Therefore the map F specified in Eq. (4.6) has a unique fixed point, and correspondingly, the BVP (2.4) has a unique solution in $C[0,1]$.

Proof Through utilizing the Cauchy-Schwarz inequality and the definition of the map $F$ described in Eq. (4.6), we get

$$
\begin{aligned}
|F v(t)-F z(t)|^{2} & \leq\left|\int_{0}^{1} G(t, s)[h(s, v(s))-h(s, z(s))] d s\right|^{2} \\
& \leq\left(\int_{0}^{1}|G(t, s)|^{2} d s\right)\left(\int_{0}^{1}|h(s, v(s))-h(s, z(s))|^{2} d s\right) \\
& \leq \eta \int_{0}^{1}\left(9+|v(s)-z(s)|^{2}\right)|v(s)-z(s)|^{2} d s .
\end{aligned}
$$

Taking the supremum over $[0,1]$ along with the definition of the metric (4.7) and a controlled function $\mathfrak{C}$, we obtain

$$
d_{\mathfrak{C}}(F v, F z) \leq \eta \mathfrak{C}(v, z) d_{\mathfrak{C}}(v, z)
$$

and the inequalities (3.2), (3.3) and (3.4) hold for any $r_{0} \in X$. Thus, it appears to follow that the map $F$ defined in Eq. (4.6) meets the criteria of Theorem 3.1 and henceforth has a unique fixed point. Hence by Lemma 4.2, the BVP (2.4) has a unique solution in $C[0,1]$. 


\section{Example}

In this section, we add an example to Theorem 3.1.

Example 5.1 Consider the following differential equation of fractional order:

$$
{ }^{c} D^{\frac{7}{2}} v(t)+h(t, v(t))=0, \quad 0<t<1,
$$

where $h$ is defined by

$$
h(t, v(t))= \begin{cases}\frac{1}{v(t)(1-v(t))}, & \text { if }-1 \leq v<1 \\ \frac{1}{v(t)(1+\nu(t))}, & \text { otherwise, }\end{cases}
$$

which is singular at $v=0$ with the boundary conditions

$$
v^{\prime \prime}(0)=v^{\prime \prime \prime}(0)=0, \quad v^{\prime}(0)=v(1)=\frac{1}{2} \int_{0}^{1} v(s) d s .
$$

The solution of the BVP provided in the example can be verified to fulfil the following integral equation:

$$
v(t)=\int_{0}^{1} G(t, s) h(s, v(s)) d s
$$

where the Green's function $G(t, s)$ is given by

$$
G(t, s)=\frac{4}{21 \Gamma\left(\frac{7}{2}\right)} \begin{cases}\left\{\frac{21}{4}+t\left(\frac{5}{2}+s\right)\right\}(1-s)^{5 / 2}-\frac{21}{4}(t-s)^{5 / 2}, & \text { if } 0 \leq s \leq t \leq 1 \\ \left\{\frac{21}{4}+t\left(\frac{5}{2}+s\right)\right\}(1-s)^{5 / 2}, & \text { if } 0 \leq t \leq s \leq 1\end{cases}
$$

Note that, in this example, $\alpha=\frac{7}{2}, \beta=\frac{1}{2}$ which fulfill the requirement (4.8), and by Lemma 4.1, we have

$$
\eta=\int_{0}^{1}|G(t, s)|^{2} d s \leq \frac{103,168}{212,625 \pi} \approx 0.15<1
$$

For the Green's function acquired in Eq. (5.2) and the related map $F$ defined by Eq. (4.6), we use the Cauchy-Schwarz inequality to derive

$$
\begin{aligned}
|F v(t)-F z(t)|^{2} & \leq\left|\int_{0}^{1} G(t, s)[h(s, v(s))-h(s, z(s))] d s\right|^{2} \\
& \leq\left(\int_{0}^{1}|G(t, s)|^{2} d s\right)\left(\int_{0}^{1}|h(s, v(s))-h(s, z(s))|^{2} d s\right) \\
& =\eta\left(\int_{0}^{1}|h(s, v(s))-h(s, z(s))|^{2} d s\right),
\end{aligned}
$$


where $\eta<1$ and

$$
\begin{aligned}
& |h(s, v(s))-h(s, z(s))| \\
& \quad= \begin{cases}\left|\frac{1}{v(s)(1-v(s))}-\frac{1}{z(s)(1-z(s) \mid}\right|, \quad \text { if } v, z \in[-1,1), \\
\left|\frac{1}{v(s)(1+v(s))}-\frac{1}{z(s)(1+z(s))}\right|, \quad \text { if } v, z \in(-\infty,-1) \cup[1,+\infty), \\
\left|\frac{1}{v(s)(1-v(s))}-\frac{1}{z(s)(1+z(s))}\right|, \quad \text { if } v \in[-1,1), z \in(-\infty,-1) \cup[1,+\infty), \\
\left|\frac{1}{v(s)(1+v(s))}-\frac{1}{z(s)(1-z(s))}\right|, \quad \text { if } v \in(-\infty,-1) \cup[1,+\infty), z \in[-1,1) .\end{cases}
\end{aligned}
$$

For $v, z \in[-1,1)$, we see that

$$
\begin{aligned}
|F v(t)-F z(t)|^{2} & \leq \eta \int_{0}^{1}\left|\frac{1}{v(s)(1-v(s))}-\frac{1}{z(s)(1-z(s))}\right|^{2} d s \\
& =\eta \int_{0}^{1}\left|\frac{(v(s))^{2}-(z(s))^{2}-(v(s)-z(s))}{v(s) z(s)(1-v(s))(1-z(s))}\right|^{2} d s \\
& =\eta \int_{0}^{1}|v(s)-z(s)|^{2}\left(\frac{(v(s)+z(s))-1)}{v(s) z(s)(1-v(s))(1-z(s))}\right)^{2} d s \\
& \leq \eta \int_{0}^{1}|v(s)-z(s)|^{2}\left(\frac{(v(s)+z(s))+1)}{v(s) z(s)}\right)^{2} d s \\
& =\eta \int_{0}^{1}|v(s)-z(s)|^{2}\left(\frac{\left.(v(s)+z(s))^{2}+2(v(s)+z(s))+1\right)}{v(s) z(s)}\right)^{2} d s \\
& \leq \eta \int_{0}^{1}|v(s)-z(s)|^{2}\left(\frac{\left.(v(s)-z(s))^{2}+4 v(s) z(s)+2(v(s)+z(s))+1\right)}{v(s) z(s)}\right)^{2} d s \\
& \leq \eta \int_{0}^{1}|v(s)-z(s)|^{2}\left(9+|v(s)-z(s)|^{2}\right) d s .
\end{aligned}
$$

By taking the supremum over $t \in[0,1]$ and taking into consideration the metric definition given in (4.7), one has

$$
d_{\mathfrak{C}}(F v, F z) \leq \eta \mathfrak{C}(v, z) d_{\mathfrak{C}}(v, z)
$$

where

$$
\mathfrak{C}(v, z)=9+\sup _{t \in[0,1]}|v(t)-z(t)|^{2}
$$

Similarly, we can prove the other cases. Therefore the map $F$ specified by (4.6) has a unique fixed point and perhaps the BVP provided in the example does have a unique solution in $C[0,1]$.

\section{Conclusion}

Following up Sevinik Adigüzel et al. [40], in this study, we dealt with problem (2.4) in the context of controlled $b$-Branciari metric type spaces, which is a stronger concept than the concept of extended-Branciari $b$-distance spaces [2] and controlled metric type spaces [33], providing a disparate approach to the existence and uniqueness of the solution. This 
method can also be used for different $\alpha$-derivative intervals. The theorem of existenceuniqueness in this study strengthens the current research as it provides quite requisites not only for positive solutions but also for any continuous solutions to the problem.

\section{Acknowledgements}

We all authors are thankful to the anonymous referees for their useful comments/suggestions. The author T. Abdeljawad would like to thank Prince Sultan University for funding this work through research group Nonlinear Analysis Methods in Applied Mathematics (NAMAM) group number RG-DES-2017-01-17. The first, second and third authors would like to thank the Management of Sri Sivasubramaniya Nadar College of Engineering, Kalavakkam-603110, for their continuous support and encouragement to carry out this research work.

Funding

Not applicable.

Availability of data and materials

Not applicable.

\section{Competing interests}

The authors declare that they have no competing interests.

\section{Authors' contributions}

All authors contributed equally and significantly to this paper. All authors have read and approved the final version of the manuscript.

\section{Author details}

${ }^{1}$ Department of Mathematics, Sri Sivasubramaniya Nadar College of Engineering, Kalavakkam, Chennai, 603 110, India.

${ }^{2}$ Department of Mathematics and General Sciences, Prince Sultan University, P.O. Box 66833, Riyadh 11586, Saudi Arabia ${ }^{3}$ Department of Medical Research, China Medical University, Taichung, Taiwan. ${ }^{4}$ Department of Computer Science and Information Engineering, Asia University, Taichung, Taiwan.

\section{Publisher's Note}

Springer Nature remains neutral with regard to jurisdictional claims in published maps and institutional affiliations.

Received: 18 August 2020 Accepted: 7 January 2021 Published online: 19 January 2021

\section{References}

1. Abdeljawad, T., Agrawal, R.P., Karapınar, E., Sumati Kumari, P.: Solutions of the nonlinear integral equation and fractional differential equation using the technique of a fixed point with a numerical experiment in extended b-metric space. Symmetry 11, 686 (2019)

2. Abdeljawad, T., Karapinar, E., Panda, S.K., Mlaiki, N.: Solutions of boundary value problems on extended-Branciari b-distance. J. Inequal. Appl. 2020, 103 (2020)

3. Agarwal, R.P., Ahmad, B.: Existence of solutions for impulsive anti-periodic boundary value problems of fractional semilinear evolution equations. Dyn. Contin. Discrete Impuls. Syst., Ser. A Math. Anal. 18, 457-470 (2011)

4. Ahmad, B., Agarwal, R.P.: On nonlocal fractional boundary value problems. Dyn. Contin. Discrete Impuls. Syst., Ser. A Math. Anal. 18, 535-544 (2011)

5. Alghamdi, M.A., Gulyaz-Ozyurt, S., Karapınar, E.: A note on extended Z-contraction. Mathematics 8, 195 (2020)

6. Alqahtani, B., Aydi, H., Karapınar, E., Rakočević, V.: A solution for Volterra fractional integral equations by hybrid contractions. Mathematics 7, 694 (2019)

7. Alsulami, H.H., Gülyaz, S., Karapınar, E., Erhan, I.M.: An Ulam stability result on quasi-b-metric-like spaces. Open Math. 2016(14), 1087-1103 (2016)

8. Bai, C.: Impulsive periodic boundary value problems for fractional differential equation involving Riemann-Liouville sequential fractional derivative. J. Math. Anal. Appl. 384, 211-231 (2011)

9. Bai, Z., Lü, H.: Positive solutions for boundary value problem of nonlinear fractional differential equation. J. Math. Anal. Appl. 311, 495-505 (2005)

10. Banach, S.: Sur les operations dans les ensembles abstract et leur applications aux equations integrals. Fundam. Math 3, 133-181 (1922)

11. Benchohra, M., Nieto, J.J., Ouahab, A.: Second-order boundary value problem with integral boundary conditions. Bound. Value Probl. 2011, Article ID 260309 (2011)

12. Cabada, A., Hamdi, Z.: Nonlinear fractional differential equations with integral boundary value conditions. Appl. Math. Comput. 228, 251-257 (2014)

13. Chifu, C., Karapınar, E., Petruşel, G.: Fixed point results in $\epsilon$-chainable complete $b$-metric spaces. Fixed Point Theory 21(2), 453-464 (2020)

14. Cichoń, M., Salem, H.A.H.: On the lack of equivalence between differential and integral forms of the Caputo-type fractional problems. J. Pseudo-Differ. Oper. Appl. (2020). https://doi.org/10.1007/s11868-020-00345-z

15. Czerwik, S.: Nonlinear set-valued contraction mappings in b-metric spaces. Atti Semin. Mat. Fis. Univ. Modena 46(2), 263-276 (1993)

16. Fabiano, N., Nikolić, N., Thenmozhi, S., Radenović, S., CYtaković, N.: Tenth order boundary value problem solution existence by fixed point theorem. J. Inequal. Appl. 2020, 166 (2020) 
17. Feng, M., Zhang, X., Ge, W.: New existence results for higher-order nonlinear fractional differential equation with integral boundary conditions. Bound. Value Probl. 2011, Article ID 720702 (2011)

18. Fulga, A., Karapınar, E., Petruşel, G.: On hybrid contractions in the context of quasi-metric spaces. Mathematics 8,675 (2020)

19. Girejko, E., Mozyrska, D., Wyrwas, M.: A sufficient condition of viability for fractional differential equations with the Caputo derivative. J. Math. Anal. Appl. 381, 146-154 (2011)

20. Gülyaz Özyurt, S.: On some $\alpha$-admissible contraction mappings on Branciari $b$-metric spaces. Adv. Theory Nonlinear Anal. Appl. 1(1), 1-13 (2017)

21. He, Y.: Existence and multiplicity of positive solutions for singular fractional differential equations with integral boundary value conditions. Adv. Differ. Equ. 2016, 31 (2016)

22. Jankowski, T.: Positive solutions for fourth-order differential equations with deviating arguments and integral boundary conditions. Nonlinear Anal. 73, 1289-1299 (2010)

23. Kalpana, G., Sumaiya Tasneem, Z.: Some fixed point results in extended hexagonal $b$-metric spaces approach to the existence of a solution to Fredholm integral equations. J. Math. Anal. 11(2), 1-17 (2020)

24. Karanar, E., Chifu, C.: Results in wt-distance over b-metric spaces. Mathematics 8, 220 (2020)

25. Karapinar, E., Abdeljawad, T., Jarad, F:: Applying new fixed point theorems on fractional and ordinary differential equations. Adv. Differ. Equ. 2019, 421 (2019). https://doi.org/10.1186/s13662-019-2354-3

26. Karapınar, E., Fulga, A., Rashid, M., Shahid, L., Aydi, H.: Large contractions on quasi-metric spaces with an application to nonlinear fractional differential-equations. Mathematics 7, 444 (2019)

27. Karapınar, E., Piri, H., Alsulami, H.H.: Fixed points of generalized F-Suzuki type contraction in complete $b$-metric spaces. Discrete Dyn. Nat. Soc. 2015, Article ID 969726 (2015)

28. Kilbas, A.A., Srivastava, H.M., Trujillo, J.J.: Theory and Applications of Fractional Differential Equations. North-Holland Math. Stud., vol. 204. Elsevier, Amsterdam (2006)

29. Kilbas, A.A., Trujillo, J.J.: Differential equations of fractional order: methods results and problems I. Appl. Anal. 78, 153-192 (2001)

30. Kilbas, A.A., Trujillo, J.J.: Differential equations of fractional order: methods results and problems II. Appl. Anal. 81 435-493 (2002)

31. Kutbi, M.A., Karapinar, E., Ahmad, J., Azam, A.: Some fixed point results for multi-valued mappings in $b$-metric spaces. J. Inequal. Appl. 2014, 126 (2014)

32. Miller, K.S., Ross, B.: An Introduction to the Fractional Calculus and Fractional Differential Equations. Wiley, New York (1993)

33. Mlaiki, N., Aydi, H., Souayah, N., Abdeljawad, T.: Controlled metric type spaces and the related contraction principle. Mathematics 6, 194 (2018)

34. Ntouyas, S.K., Wang, G., Zhang, L.: Positive solutions of arbitrary order nonlinear fractional differential equations with advanced arguments. Opusc. Math. 31, 433-442 (2011)

35. Podlubny, l.: Fractional Differential Equations, Mathematics in Science and Engineering. Academic Press, New York (1999)

36. Salem, H.A.H.: Fractional order boundary value problem with integral boundary conditions involving Pettis integral. Acta Math. Sci. Ser. B Engl. Ed. 31(2), 661-672 (2011)

37. Salem, H.A.H.: On functions without pseudo derivatives having fractional pseudo derivatives. Quaest. Math. 42(9), 1237-1252 (2019)

38. Samko, S.G., Kilbas, A.A., Marichev, O.I.: Fractional Integrals and Derivatives. Theory and Applications. Gordon \& Breach, Yverdon (1993)

39. Sarwar, M., Zada, M.B., Radenović, S.: Rational type inequality with applications to Volterra-Hammerstein nonlinear integral equations. Int. J. Nonlinear Sci. Numer. Simul. (2020). https://doi.org/10.1515/ijnsns-2018-0367

40. Sevinik Adigüzel, R., Aksoy, U., Karapinar, E., Erhan, I.M.: On the solution of a boundary value problem associated with a fractional differential equation. Math. Methods Appl. Sci. (2020). https://doi.org/10.1002/mma.6652

41. Sumaiya Tasneem, Z., Kalpana, G., Abdeljawad, T.: Controlled $b$-Branciari metric type spaces and related fixed point theorems with applications. Filomat. To appear

42. Todorčević, V: Subharmonic behavior and quasiconformal mappings. Anal. Math. Phys. 9(3), 1211-1225 (2019)

43. Todorčević, V.: Harmonic Quasiconformal Mappings and Hyperbolic Type Metrics. Springer, Cham (2019)

44. Wang, G.: Boundary value problems for systems of nonlinear integro-differential equations with deviating arguments. J. Comput. Appl. Math. 234, 1356-1363 (2010)

45. Wang, G., Ahmad, B., Zhang, L.: Impulsive anti-periodic boundary value problem for nonlinear differential equations of fractional order. Nonlinear Anal. 74, 792-804 (2011)

46. Wang, G., Song, G., Zhang, L.: Integral boundary value problems for first order integro-differential equations with deviating arguments. J. Comput. Appl. Math. 225, 602-611 (2009)

47. Zhang, S.: The existence of a positive solution for a nonlinear fractional differential equation. J. Math. Anal. Appl. 252, 804-812 (2000)

48. Zhang, X.M., Feng, M.Q., Ge, W.G.: Existence result of second-order differential equations with integral boundary conditions at resonance. J. Math. Anal. Appl. 353, 311-319 (2009) 